\title{
A Multimode Responsive Aptasensor for Adenosine Detection
}

\author{
Na Zhao, ${ }^{1}$ Dan Zhao, ${ }^{1}$ Li-ping Xu, ${ }^{1}$ Linfeng Chen, ${ }^{2}$ Yongqiang Wen, ${ }^{1}$ and Xueji Zhang ${ }^{1}$ \\ ${ }^{1}$ Research Center for Bioengineering \& Sensing Technology, School of Chemistry \& Biological Engineering, \\ University of Science and Technology Beijing, Beijing 100083, China \\ ${ }^{2}$ Beijing National Laboratory for Molecular Sciences (BNLMS), Institute of Chemistry, Chinese Academy of Sciences, \\ Beijing 100190, China \\ Correspondence should be addressed to Yongqiang Wen; wyq-wen@ustb.edu.cn
}

Received 10 October 2014; Accepted 29 November 2014; Published 21 December 2014

Academic Editor: William W. Yu

Copyright (c) $2014 \mathrm{Na}$ Zhao et al. This is an open access article distributed under the Creative Commons Attribution License, which permits unrestricted use, distribution, and reproduction in any medium, provided the original work is properly cited.

\begin{abstract}
We report a novel multimode detection aptasensor with three signal responses (i.e., fluorescence recovery, enhanced Raman signal, and color change). The presence of adenosine induces the conformational switch of the adenosine aptamer (Apt), forming adenosine-aptamer complex and releasing quantum dots (QDs) from AuNPs, resulting in the recovered fluorescence, the enhanced Raman signal, and color change of the solution. The multimode signal recognition is potentially advantageous in improving the precision and reliability of the detection in complex environments compared to the conventional single-mode sensing system. The multimode detection strategy opens up a new possibility in sensing and quantifying more other target molecules.
\end{abstract}

\section{Introduction}

As an endogenous purine nucleoside occurring in all cells of the human system, adenosine performs extremely important signaling functions in the cardiovascular, nervous, and immune systems. Therefore, the monitoring of adenosine is of great importance in disease diagnosis and clinical medicine [1]. Current approaches including capillary electrophoresis (CE) [2], high-performance liquid chromatography (HPLC) [3], fluorescence resonance-energy transfer (FRET) [4-7], and colorimetry $[8,9]$ have been demonstrated for quantitative detection of adenosine. For example, assays based on FRET using an adenosine-aptamer (Apt) modified with a fluorophore (donor) and a quencher (acceptor) allow the sensing of adenosine under intracellular or extracellular conditions $[4,6]$. Liu and Lu employed a single-strand linker DNA that contained the aptamer sequence to induce the aggregation of AuNPs; the addition of adenosine dissembled the aggregated AuNPs and resulted in a purple-to-red color change [10]. Although these assays have successfully detected adenosine, one of their disadvantages is that only one kind of phenomenon or signal was applied to determine the presence of adenosine. These single-mode techniques are susceptible to the complex environments, which might be interfered in the detection process, even resulting in false results. To develop a multicomponent sensor that can yield diverse signals in response to an analyte in one pot still remains a challenging task.

Over the past 20 years, DNA molecules have been utilized to design a variety of nanoscale structures and devices for a variety of applications due to the unique molecular recognition capabilities and structural features [11-13]. In parallel, not only do they function as precise assembly of specific nanostructure, but also specific DNA sequence can do catalytic functions like protein enzymes (DNAzymes) and specific binding functions like antibodies (i.e., aptamers). Aptamers, which have highly specific molecular recognition merits, have played an important role in aptamer-based biosensors of molecular diagnostics and therapeutics $[14,15]$. The development of aptamers opens a new field for highly sensitive and simple detection of small molecules due to the unprecedented advantages of aptamers such as high affinity and specificity to a wide range of target molecules, including ions, small organic molecules, proteins, and cells [15-17].

Taking advantage of the highly specific binding abilities of aptamers, the characteristics of flexible designability, and the highly precise positioning and control capability of DNA molecules, we herein report a multimode sensing system 


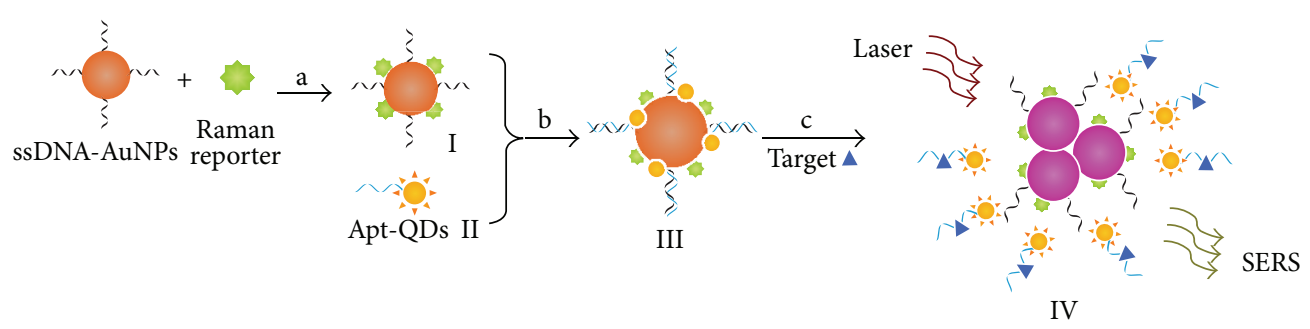

SCHEME 1: Schematic representation of the multimode aptasensor for adenosine detection.

that can give three signals in one pot in response to adenosine simultaneously, namely, fluorescence recovery, enhanced Raman signal, and color change of the solution. Normally, the triple-mode recognition to one target has the potential to make the experiment results more reliable compared to single-response biosensors. This fundamental concept of multimode signal recognition opens up more opportunities for designing more reliable sensors systems in nanomedicine.

\section{Materials and Methods}

2.1. Materials. $\mathrm{HAuCl}_{4}$, trisodium citrate, cadmium oxide, zinc oxide selenium, sulfur, trioctylphosphine oxide (TOPO), tributylphosphine (TBP), 1-octadecene (ODE), oleic acid (OA), 3-mercaptopropionic acid (MPA), N-(3-dimethylaminopropyl)-N-ethylcarbodiimide (EDC), N-hydroxysuccinimide (NHS), and 4-aminothiophenol (PATP) were purchased from Sigma-Aldrich (USA). Adenosine was obtained from Solarbio (Beijing, China). CdSe QDs and AuNPs were synthesized by us according to the literatures $[18,19]$. The oligonucleotides with the following sequences $5^{\prime}$ TGGAAGGAGG-CGTTATGAGGGGGTCCAGTCAC-C ${ }^{-}$ $\mathrm{NH}_{2}-3^{\prime}$ and $5^{\prime}-\mathrm{SH}-\mathrm{C}_{6}$-GT-GACTGGACCC- $3{ }^{\prime}$ were obtained and were HPLC-purified at Sangon Biotechnology Inc. (Shanghai, China). All other materials were of analytical grade and were used as received. All buffers performed with ultrapure Milli-Q water (resistance $>18 \mathrm{M} \Omega \cdot \mathrm{cm}^{-1}$ ) were used in all experiments.

2.2. Apparatus. The morphology and the size of AuNPs were obtained by performing transmission electron microscopy (TEM, JEM-2100F). UV-Vis absorption spectra of all samples at every step of the experiments were measured with a UV$1800 \mathrm{UV}-\mathrm{Vis}$ spectrometer, in the 500 and $700 \mathrm{~nm}$ wavelength range. The fluorescence emission spectra were measured on a F-4500 luminescence spectrometer equipped with a xenon lamp as a light source. Two cuvettes of $2 \mathrm{~mm}$ path length quartz were used to measure the fluorescence and absorption spectra separately. Raman measurements were conducted in inVia-Reflex (UK) Raman spectrometer at an excitation laser of $633 \mathrm{~nm}$. All experiments were carried out at room temperature under ambient conditions.

2.3. Preparation of Single-Stranded DNA (ssDNA) and Raman Reporter Functionalized AuNPs (Scheme 1, I). AuNPs, $13 \mathrm{~nm}$ in diameter, were synthesized by classical Turkevich-Frens method [19]. Briefly, $100 \mathrm{~mL} \mathrm{H}_{2} \mathrm{O}$ and $2 \mathrm{~mL} 1 \% \mathrm{HAuCl}_{4}$ were added into $250 \mathrm{~mL}$ three-neck flask and heated under vigorous agitation. Then, $8 \mathrm{~mL}$ freshly prepared $1 \%$ sodium citrate solution was rapidly added to the boiling $\mathrm{HAuCl}_{4}$ solution. After 30 minutes of refluxing, the solution was cooled to room temperature. The concentration was estimated by Beer-Lambert law, based on an extinction coefficient of 2.43 $\times 10^{8} \mathrm{M}^{-1} \mathrm{~cm}^{-1}$ at $\lambda=520 \mathrm{~nm}$ [6]. The as-prepared stabilized $13 \mathrm{~nm}$ AuNPs were stored at $4^{\circ} \mathrm{C}$. For ssDNA-AuNPs, thiolated DNA oligonucleotides $\left(5^{\prime}-\mathrm{SH}-\mathrm{C}_{6}\right.$-GTGACTGGACCC $-3^{\prime}, 70 \mu \mathrm{L}, 10 \mu \mathrm{M}$ ) were added to $200 \mu \mathrm{L}$ AuNPs (16 nM) aqueous solution. After overnight incubation, the Raman reporters (PATP ethanol solutions, $10 \mu \mathrm{L}, 0.03 \mu \mathrm{M}$ ) were added into as-prepared AuNPs solutions for $24 \mathrm{~h}$. After that, the AuNPs conjugates labeled by ssDNA and Raman reporters were "aged" in $0.1 \mathrm{M}$ PBS solution ( $\mathrm{pH}$ 7.4) for $24 \mathrm{~h}$ at ambient temperature [4]. Unconjugated oligonucleotides were removed by centrifugation at $12000 \mathrm{rpm}$ for $15 \mathrm{~min}$ and the red oily precipitate was washed, recentrifuged, and then dispersed in $200 \mu \mathrm{L}$ Tris- $\mathrm{HCl}(20 \mathrm{mM}, \mathrm{pH} 7.4)$ at $4^{\circ} \mathrm{C}$ for future use.

\subsection{Preparation of Apt Functionalized CdSe/ZnS QDs} (Scheme 1, II). CdSe/ZnS QDs were synthesized by the well-established method with MPA modification [18]. The concentration of CdSe/ZnS QDs wascalculated by classical Xiaogang Peng method [20]. The COOH-functionalized QDs $(0.04 \mathrm{nmol})$ were activated with $60 \mathrm{nmol}$ EDC and $60 \mathrm{nmol}$ NHS for $30 \mathrm{~min}$, followed by incubating with Apt (5'-TG-GAAGGAGGCGTTATGAGGGGGTCCAGTCAC$\left.\mathrm{C}_{6}-\mathrm{NH}_{2}-3^{\prime}\right)$ for $12 \mathrm{~h}$. After that, aqueous $\mathrm{NaCl}(40 \mu \mathrm{L}, 1 \mathrm{M})$ and $\mathrm{CH}_{3} \mathrm{CH}_{2} \mathrm{OH}(20 \mu \mathrm{L})$ were added; the mixture was then purified in a centrifuge at $8000 \mathrm{rpm}$ for $5 \mathrm{~min}$. The Apt-ODs were then dispersed in Tris- $\mathrm{HCl}$ buffer $(13.6 \mu \mathrm{L}, 20 \mathrm{mM}, \mathrm{pH}$ 7.4) $[6]$.

2.5. Preparation of QDs-AuNPs Probes (Scheme 1, III) and Adenosine Detection. In order to ensure the stability and accuracy of the experiment result, the DNA strands on Apt-QDs (Scheme 1, II) and ssDNA-AuNPs (Scheme 1, I) conjugates should form double-strand DNA conjugate as much as possible. We mixed Apt-QDs and ssDNA-AuNPs conjugates in a series of molar ratio and incubated at $70^{\circ} \mathrm{C}$ for $10 \mathrm{~min}$, and then cooled down to room temperature to ensure the maximization of hybridization. Fluorescence quenching efficiency was used to detect the hybridization 
efficiency of the two DNA strands. By performing a series of experiments, the optimum molar ratio of Apt-QDs and ssDNA-AuNPs conjugates was determined as $12: 1$. Then, the QDs-AuNPs probes solution was centrifuged and washed once with an equal volume of PBS ( $\mathrm{pH} 7.4$ ) and finally resuspended in PBS ( $1 \mathrm{~mL}, \mathrm{pH} 7.4)$. For analyte detection, adenosine of various concentrations was added to the QDsAuNPs probes solution and incubated for $30 \mathrm{~min}$ at room temperature.

\section{Results and Discussion}

The principle of FERT biosensor is shown in Scheme 1. In the first step, the AuNPs were modified with the thiol-DNA, which could be easily absorbed onto citrate coated AuNPs and stabilize the AuNPs in salt or buffer solution. Then the ssDNA-attached AuNPs (ssDNA-AuNPs), in which the DNA sequence was partially complementary with the Apt sequence, were labeled with Raman reporter through the Au-S bonds. The process was shown in Scheme la. Subsequently, the AuNPs conjugates (Scheme 1, I) were hybridized with the Apt-QDs (Scheme 1, II), forming the QDs-AuNPs probes (Scheme 1, III) with red in high salt concentration (Scheme 1b). In this conformation, the nanoprobe only exhibited small background fluorescence due to the fluorescence transferring from the QDs to the AuNPs when they are in close proximity.

FRET between QDs as donor and AuNPs as acceptor was used for detection of adenosine. As an alternative developed inorganic fluorescent material, QDs have unique virtues compared to the traditional fluorophores, such as size tunable photoluminescence, high quantum yields, narrowsize tunable spectra, and low photobleaching [21, 22]. Gold nanoparticles have been of great interest because of the superior quenching effect and the color change associated with its states. Thiolated molecules (such as ssDNA and Raman reporter) especially can be easily attached on the AuNPs surface through strong gold-sulphur covalent reaction [23].

When the adenosine is present, the aptamer-target binding displaced the Watson-Crick binding between the Apt and the linker DNA sequence on QDs, which induces the conformational switch of the Apt from loose random coil to a Gquadruplex structure, which disassembles the original duplex and the QDs were separated from the AuNPs, affording fluorescence "turn-on" state due to the far distance between donor and acceptor (Scheme 1c). The relationship between the increase of fluorescence intensity and the adenosine concentration provides the basis for the quantitation of adenosine. Simultaneously, the ssDNA-AuNPs were aggregated (Scheme 1, IV) because of the different stabilities of ssDNAAuNPs and double-stranded (ds) DNA-attached AuNPs (dsDNA-AuNPs) in a high salt concentration, leading to a corresponding color change from red to purple (Scheme 1c). This process leads to redistribution of the ssDNA-AuNPs and a great enhancement of the EM field at a specific position around the AuNPs surface (called "hot spots"). The "hot spots" were formed between the junctions of the AuNPs aggregates (Scheme 1, IV), giving rise to enhanced Raman

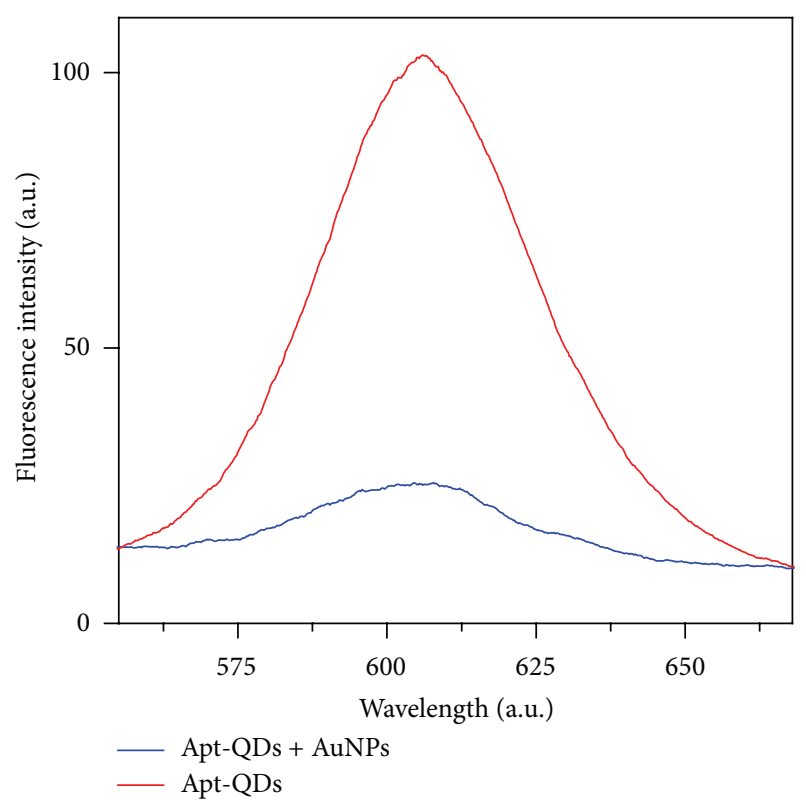

FIGURE 1: Fluorescence spectra of QDs in the absence and the presence of the AuNPs.

scattering signal under the light excitation at wavelength of $633 \mathrm{~nm}$ (Scheme 1c).

3.1. Fluorescence Quenching Efficiency by the AuNPs. The fluorescence of the QDs in the QDs-AuNPs probes was highly quenched by the AuNPs through the FRET. Because the quenching efficiency within the conjugates is one of the key factors for the sensitivity of adenosine detection, we first determined the quenching efficiency of our system by comparing the fluorescence signal of both the QDs and QDsAuNPs probes. Fluorescence spectra of QDs donor in the absence and the presence of the acceptor AuNPs were shown in Figure 1, respectively. Quenching efficiency was calculated by [24]

$$
\text { Quenching efficiency }=\frac{F_{0}-F}{F_{0}} \text {. }
$$

In (1), $F_{0}, F$ are the fluorescence intensity of QDs at $606 \mathrm{~nm}$ before and after addition of ssDNA-AuNPs conjugates, respectively. Maximal quenching efficiency $75 \%$ was observed in Figure 1 with 12:1 molar ratio of Apt-QDs to ssDNA-AuNPs. The result suggests that FRET indeed occurred and ssDNA-AuNPs are highly effective to quench the QDs fluorescence.

3.2. Effect of the Ionic Strength to the QDs-AuNPs and ssDNA-AuNPs Systems. AuNPs have been widely applied as colorimetric reporter in biomolecular detection [25-27]. The change in color of the dispersed AuNPs (red) and aggregated AuNPs (purple) can be easily read with the naked eye. Li and Rothberg found that ss-DNA and ds-DNA have different binding affinities to negatively charged AuNPs, thus resulting 


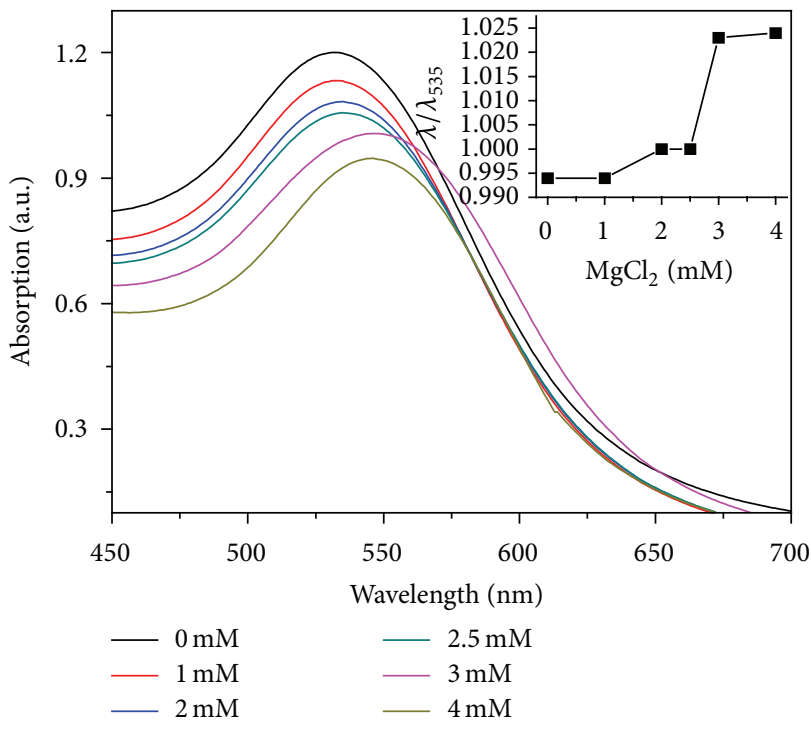

(a)

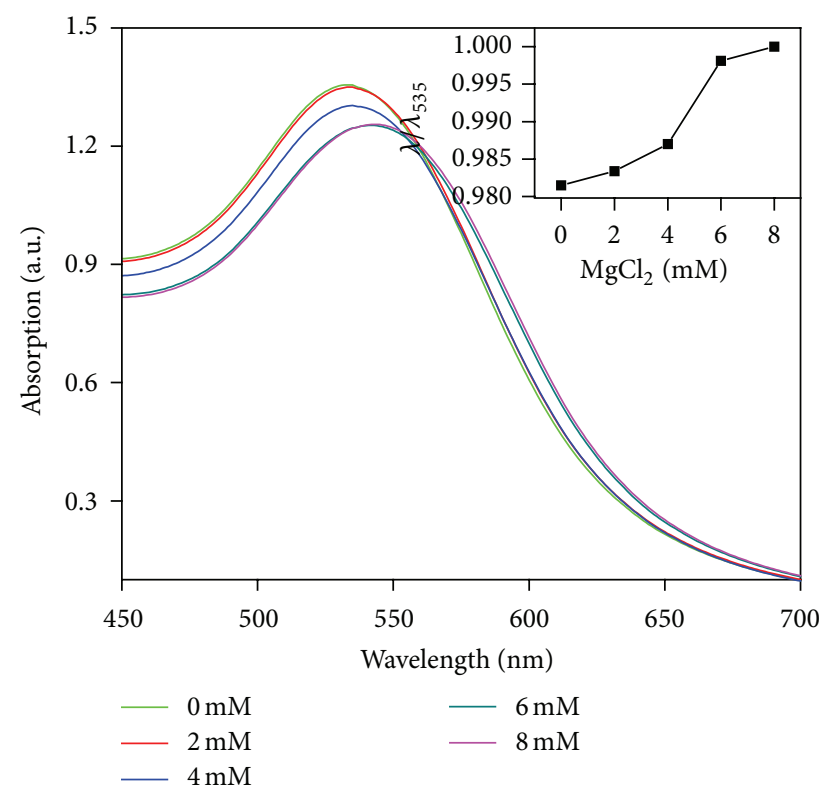

(b)

Figure 2: UV-Vis spectra of ssDNA-AuNPs and QDs-AuNPs solutions in the presence of $\mathrm{MgCl}_{2}$ at different concentrations. The concentrations of $\mathrm{MgCl}_{2}$ were $0,1,2,2.5$, and $3 \mathrm{mM}$ for ssDNA-AuNPs probes (a) and 0, 2, 4, 6, and $8 \mathrm{mM}$ for QDs-AuNPs probes (b). Inset: the plot of the wavelength ratio $\left(\lambda / \lambda_{535}\right)$ versus the increasing concentrations of $\mathrm{MgCl}_{2}$ of the same data, respectively.

in the different stabilities of ssDNA-AuNPs and dsDNAAuNPs in a high salt concentration [28].

Based on this principle, several colorimetric assays have been developed. For example, Sato and coworkers demonstrated a colorimetric detection of DNA by using the different stabilities of ssDNA-AuNPs before and after the hybridization with the complementary ssDNA. Surprisingly, they found that fully matched dsDNA-AuNPs had a lower stability than ssDNA-AuNPs [29]. On the basis of these studies, Zhao and coworkers found that dsDNA-AuNPs, which are not completely paired, were much more stable than ssDNA-AuNPs [30]. In order to determine the stabilities of ssDNA-AuNPs and QDs-AuNPs, following experiments were performed to detect the different salt tolerance of the two systems.

At a situation of $100 \mathrm{mM} \mathrm{NaCl}(\mathrm{pH}=7.4)$, a different amount of $\mathrm{MgCl}_{2}$ was slowly added into the ssDNAAuNPs and QDs-AuNPs solutions independently. As shown in Figure 2, when the concentration of $\mathrm{MgCl}_{2}$ of the two systems was $3 \mathrm{mM}$ and $8 \mathrm{mM}$, respectively, the UV absorption redshifts significantly. The redshift of the UV absorption was induced by the aggregation of AuNPs, which confirmed the fact that partially complementary dsDNA-AuNPs possessed higher salt tolerance than ssDNA-AuNPs. In subsequent experiments, the reaction systems were maintained $4 \mathrm{mM}$ $\mathrm{MgCl}_{2}$. At that situation QDs-AuNPs systems were stable whereas ssDNA-AuNPs were not.

3.3. Adenosine Substance Detection. Surface-enhanced Raman scattering (SERS) is an ultrasensitive vibrational spectroscopic technique that has long been an alternative tool for biosensing because of its unique features, such as hyperfine resolution ability, noninvasive advantage, and relaxed testing conditions [31-33]. It is acknowledged that a remarkable surface enhancement Raman scattering effect can be generated at the nanoscale junctions and interstices in interacting metal nanostructures such as nanoparticles dimmers or aggregates. Usually, an enhanced signal detected at the junctions between nanoparticles of the aggregates is $2-40$ times stronger than that obtained at an isolated nanoparticle due to the generation of the "hot spots" [34]. In our work, the "hot spots" were formed between the aggregated AuNPs after the addition of adenosine, leading to the SERS signal enhancement.

Under optimized conditions, the analytical performance of the multimode aptasensor for adenosine detection was investigated. In the QDs-AuNPs conformation, the fluorescence was efficiently quenched by AuNPs due to close donor and acceptor distance. Upon the addition of adenosine, the constrained conformation was turned on in response to the adenosine binding with its aptamer; the QDs were separated from the AuNPs, which led to a significant fluorescence increase in the emission at $606 \mathrm{~nm}$. This adenosine binding process resulted in the aggregation of the ssDNA-AuNPs in high salt solution with concomitant red-to-purple color change and enhanced Raman scattering signal. Figure 3(a) shows the fluorescence spectra of the QDs-AuNPs probes after adding various concentrations of adenosine. Sequential increases in the fluorescence emission spectrum of QDs were observed when fixed volumes of QDs-AuNPs conjugates $(200 \mu \mathrm{L})$ were incubated with increasing adenosine concentrations. As shown, the fluorescence intensity was proportionally increased according to the concentrations of adenosine in a range of 0 to $60 \mu \mathrm{M}$. Furthermore, a linear 


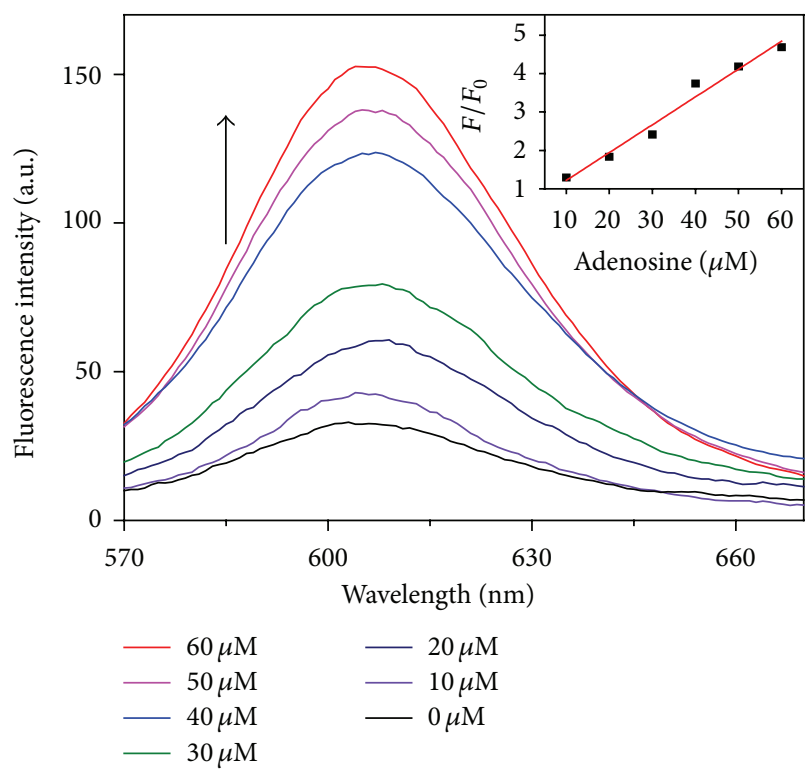

(a)

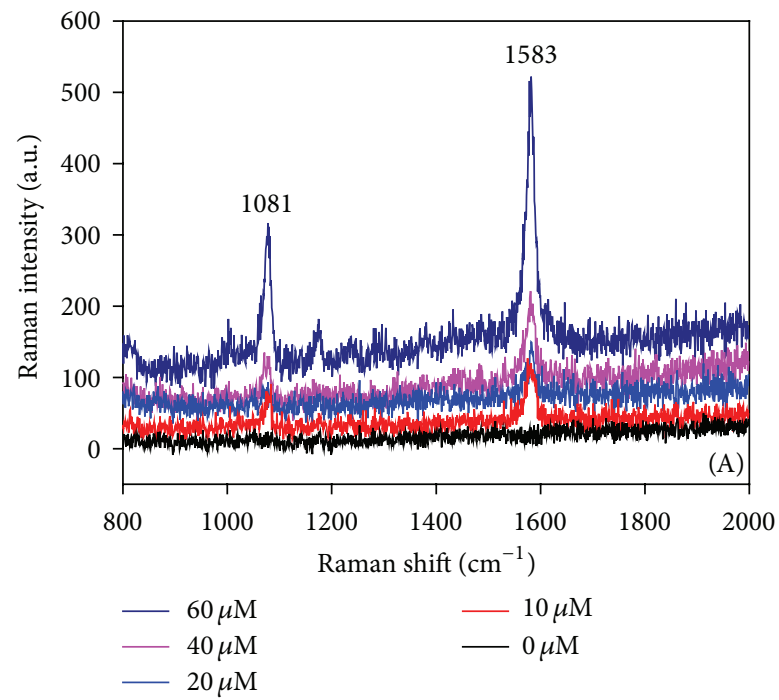

(c)

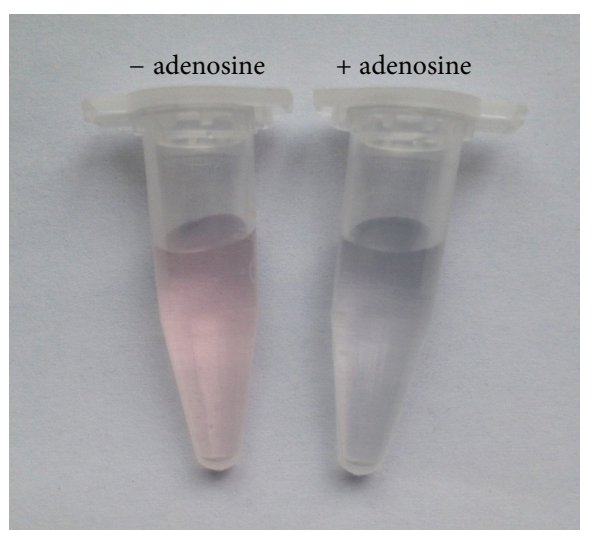

(b)

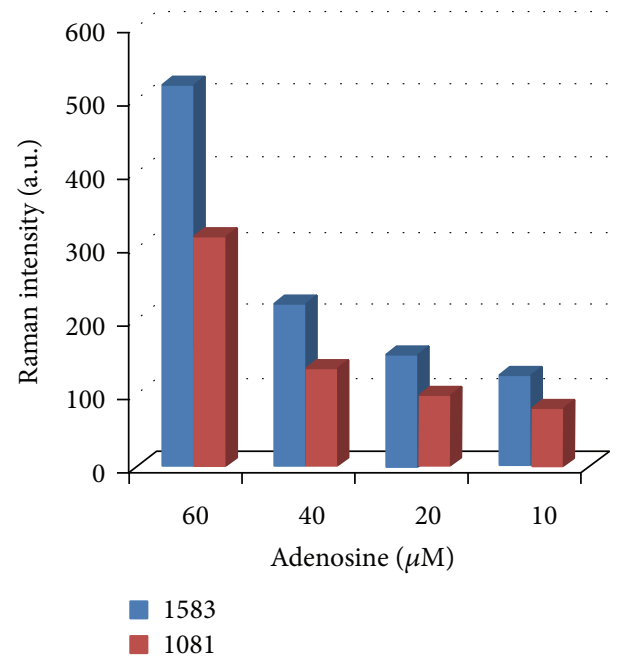

(d)

FIGURE 3: (a) Fluorescence spectra depending on adenosine concentrations from 0 to $60 \mu \mathrm{M}$. (Inset: the plot of the fluorescence ratio $\left(F / F_{0}\right)$ versus the increasing concentrations of adenosine of the same data.) (b) Photograph showing colorimetric responses of detection system in the presence of $0 \mu \mathrm{M}$ and $40 \mu \mathrm{M}$ adenosine. (c) Raman spectra obtained from the QDs-AuNPs probe with various concentrations of adenosine. (d) Raman intensity at peak of $1081 \mathrm{~cm}^{-1}$ and $1583 \mathrm{~cm}^{-1}$ of the QDs-AuNPs probe incubation with different concentrations of adenosine.

range of the fluorescence response versus the $F / F_{0}$ of the same data was also shown in Figure 3(a). The peaks at 1081 and $1583 \mathrm{~cm}^{-1}$ of the Raman spectra were the characteristics of PATP molecules, which was observed upon the addition of adenosine at various concentrations in Figure 3(c). As shown in Figures 3(c) and 3(d), the intensity of the Raman peaks increased with the increase of the adenosine concentrations. The SERS response became saturated when the adenosine concentration reached $70 \mu \mathrm{M}$ (data not shown). Therefore, the range of the SERS response was $10 \mu \mathrm{M}$ to $60 \mu \mathrm{M}$ as shown in Figure 3(c). On the contrary, no evident PATP Raman peak associated with AuNPs was observed in the absence of adenosine, which was consistent with the result in Figure 3(c)(A).
Correspondingly, the color change of the solution further confirmed the aggregation of the AuNPs, as shown in Figure 3(b). Thus, three signals were observed simultaneously within 30 minutes upon the addition of the target molecules. The general concept of multimode detection in one pot will be of significant importance in improving reliability of the analysis.

\section{Conclusions}

In summary, taking advantage of the highly specific binding abilities of aptamers, conformational flexibility of the DNA constituents, we established a system that can give three 
signal responses to adenosine in one pot. The design relies on the structure-switching properties of aptamers upon binding to their target molecules. Using Apt-QDs as the molecular recognition element and the Raman molecule labeled ssDNA-AuNPs as the signaling probe, this triplemode response aptasensor reported here opens up a new possibility of rapid, easy, and reliable detection of adenosine from more complex environments. Furthermore, our strategy provides a feasible platform for the detection of more other biomolecules owing to the high affinity and specificity of aptamers to a wide range of targets molecules. The innovative approach also holds promising potential for broad applications in the environmental monitoring, food safety, and clinical applications. Further detailed quantitative research is worth being carried out.

\section{Conflict of Interests}

The authors declare that there is no conflict of interests regarding the publication of this paper.

\section{Authors' Contribution}

Na Zhao and Dan Zhao contributed equally to this work.

\section{Acknowledgments}

The authors would like to thank the NSFC (21171019, 21073203, 51373023, 21275017, 21127007, and 21103009), the Fundamental Research Funds for the Central Universities and NCET-11-0584, Beijing Natural Science Foundation (2122038), and Educational Ministry New Century Excellent Talents Support Project (no. BMU20110270).

\section{References}

[1] V. Vallon, B. Mühlbauer, and H. Osswald, "Adenosine and kidney function," Physiological Reviews, vol. 86, no. 3, pp. 901940, 2006.

[2] H. Fang, M. L. Pajski, A. E. Ross, and B. J. Venton, "Quantitation of dopamine, serotonin and adenosine content in a tissue punch from a brain slice using capillary electrophoresis with fast-scan cyclic voltammetry detection," Analytical Methods, vol. 5, no. 11, pp. 2704-2711, 2013.

[3] S. Prado, A. Villamarín, and I. Ibarguren, "Simultaneous determination of adenosine and related purines in tissues and hemolymph of mussel by HPLC," Journal of Liquid Chromatography and Related Technologies, vol. 36, no. 4, pp. 470-485, 2013.

[4] J. Zhang, L. Wang, H. Zhang, F. Boey, S. Song, and C. Fan, "Aptamer-based multicolor fluorescent gold nanoprobes for multiplex detection in homogeneous solution," Small, vol. 6, no. 2, pp. 201-204, 2010.

[5] W. Qiang, H. Liu, W. Li, X. Chen, and D. Xu, "Label-free detection of adenosine based on fluorescence resonance energy transfer between fluorescent silica nanoparticles and unmodified gold nanoparticles," Analytica Chimica Acta, vol. 828, pp. 92-98, 2014.

[6] Y. S. Kim and J. Jurng, "Gold nanoparticle-based homogeneous fluorescent aptasensor for multiplex detection," Analyst, vol. 136, no. 18, pp. 3720-3724, 2011.
[7] M. Zhang, S.-M. Guo, Y.-R. Li, P. Zuo, and B.-C. Ye, "A labelfree fluorescent molecular beacon based on DNA-templated silver nanoclusters for detection of adenosine and adenosine deaminase," Chemical Communications, vol. 48, no. 44, pp. 5488-5490, 2012.

[8] J. Liu, H. L. Jung, and Y. Lu, "Quantum dot encoding of aptamerlinked nanostructures for one-pot simultaneous detection of multiple analytes," Analytical Chemistry, vol. 79, no. 11, pp. 41204125, 2007.

[9] Y. Yi, Y. Huang, G. Zhu et al., "A colorimetric and fluorescence sensing platform for two analytes in homogenous solution based on aptamer-modified gold nanoparticles," Analytical Methods, vol. 5, no. 10, pp. 2477-2484, 2013.

[10] J. Liu and Y. Lu, "Preparation of aptamer-linked gold nanoparticle purple aggregates for colorimetric sensing of analytes," Nature Protocols, vol. 1, no. 1, pp. 246-252, 2006.

[11] H. Liang, X. B. Zhang, Y. Lv et al., "Functional DNA-containing nanomaterials: cellular applications in biosensing, imaging, and targeted therapy," Accounts of Chemical Research, vol. 47, no. 6, pp. 1891-1901, 2014.

[12] A. V. Pinheiro, D. Han, W. M. Shih, and H. Yan, "Challenges and opportunities for structural DNA nanotechnology," Nature Nanotechnology, vol. 6, no. 12, pp. 763-772, 2011.

[13] J. Wang, "Nanomaterial-based amplified transduction of biomolecular interactions," Small, vol. 1, no. 11, pp. 1036-1043, 2005.

[14] J. G. Bruno, "A review of therapeutic aptamer conjugates with emphasis on new approaches," Pharmaceuticals, vol. 6, no. 3, pp. 340-357, 2013.

[15] N. K. Navani and Y. Li, "Nucleic acid aptamers and enzymes as sensors," Current Opinion in Chemical Biology, vol. 10, no. 3, pp. 272-281, 2006.

[16] H. Wei, B. Li, J. Li, S. Dong, and E. Wang, "DNAzyme-based colorimetric sensing of lead $\left(\mathrm{Pb}^{2+}\right)$ using unmodified gold nanoparticle probes," Nanotechnology, vol. 19, no. 9, Article ID 095501, 2008.

[17] V. Pavlov, Y. Xiao, B. Shlyahovsky, and I. Willner, "Aptamerfunctionalized Au nanoparticles for the amplified optical detection of thrombin," Journal of the American Chemical Society, vol. 126, no. 38, pp. 11768-11769, 2004.

[18] J. J. Li, Y. A. Wang, W. Guo et al., "Large-scale synthesis of nearly monodisperse CdSe/CdS core/shell nanocrystals using air-stable reagents via successive ion layer adsorption and reaction," Journal of the American Chemical Society, vol. 125, no. 41, pp. 12567-12575, 2003.

[19] K. C. Grabar, R. G. Freeman, M. B. Hommer, and M. J. Natan, "Preparation and characterization of Au colloid monolayers," Analytical Chemistry, vol. 67, no. 4, pp. 735-743, 1995.

[20] W. W. Yu, L. Qu, W. Guo, and X. Peng, "Experimental determination of the extinction coefficient of CdTe, CdSe, and CdS nanocrystals," Chemistry of Materials, vol. 15, no. 14, pp. 28542860, 2003.

[21] J. Liu, Y. Liu, X. Yang et al., "Exciton energy transfer-based fluorescent sensing through aptamer-programmed self-assembly of quantum dots," Analytical Chemistry, vol. 85, no. 22, pp. 1112111128, 2013.

[22] E. Petryayeva, W. R. Algar, and I. L. Medintz, "Quantum dots in bioanalysis: a review of applications across various platforms for fluorescence spectroscopy and imaging," Applied Spectroscopy, vol. 67, no. 3, pp. 215-252, 2013. 
[23] K. Saha, S. S. Agasti, C. Kim, X. Li, and V. M. Rotello, "Gold nanoparticles in chemical and biological sensing," Chemical Reviews, vol. 112, no. 5, pp. 2739-2779, 2012.

[24] T. Kang, H. C. Kim, S.-W. Joo et al., "Optimization of energy transfer between quantum dots and gold nanoparticles in headto-head configuration for detection of fusion gene," Sensors and Actuators B: Chemical, vol. 188, pp. 729-734, 2013.

[25] D. Liu, Z. Wang, and X. Jiang, "Gold nanoparticles for the colorimetric and fluorescent detection of ions and small organic molecules," Nanoscale, vol. 3, no. 4, pp. 1421-1433, 2011.

[26] F. Xia, X. Zuo, R. Yang et al., "Colorimetric detection of DNA, small molecules, proteins, and ions using unmodified gold nanoparticles and conjugated polyelectrolytes," Proceedings of the National Academy of Sciences of the United States of America, vol. 107, no. 24, pp. 10837-10841, 2010.

[27] Y. He, S. Zhang, X. Zhang et al., "Ultrasensitive nucleic acid biosensor based on enzyme-gold nanoparticle dual label and lateral flow strip biosensor," Biosensors and Bioelectronics, vol. 26, no. 5, pp. 2018-2024, 2011.

[28] H. Li and L. Rothberg, "Colorimetric detection of DNA sequences based on electrostatic interactions with unmodified gold nanoparticles," Proceedings of the National Academy of Sciences of the United States of America, vol. 101, no. 39, pp. 14036-14039, 2004.

[29] K. Sato, K. Hosokawa, and M. Maeda, "Rapid aggregation of gold nanoparticles induced by non-cross-linking DNA hybridization," Journal of the American Chemical Society, vol. 125, no. 27, pp. 8102-8103, 2003.

[30] W. Zhao, W. Chiuman, M. A. Brook, and Y. Li, "Simple and rapid colorimetric biosensors based on DNA aptamer and noncrosslinking gold nanoparticle aggregation," ChemBioChem, vol. 8, no. 7, pp. 727-731, 2007.

[31] Y. Wang, B. Yan, and L. Chen, "SERS Tags: novel optical nanoprobes for bioanalysis," Chemical Reviews, vol. 113, no. 3, pp. 1391-1428, 2013.

[32] M. Li, J. Zhang, S. Suri, L. J. Sooter, D. Ma, and N. Wu, “Detection of adenosine triphosphate with an aptamer biosensor based on surface-enhanced Raman scattering," Analytical Chemistry, vol. 84, no. 6, pp. 2837-2842, 2012.

[33] S. Wang, L.-P. Xu, Y. Wen, H. Du, S. Wang, and X. Zhang, "Space-confined fabrication of silver nanodendrites and their enhanced SERS activity," Nanoscale, vol. 5, no. 10, pp. 42844290, 2013.

[34] F. Svedberg, Z. Li, H. Xu, and M. Käll, "Creating hot nanoparticle pairs for surface-enhanced Raman spectroscopy through optical manipulation," Nano Letters, vol. 6, no. 12, pp. 26392641, 2006. 

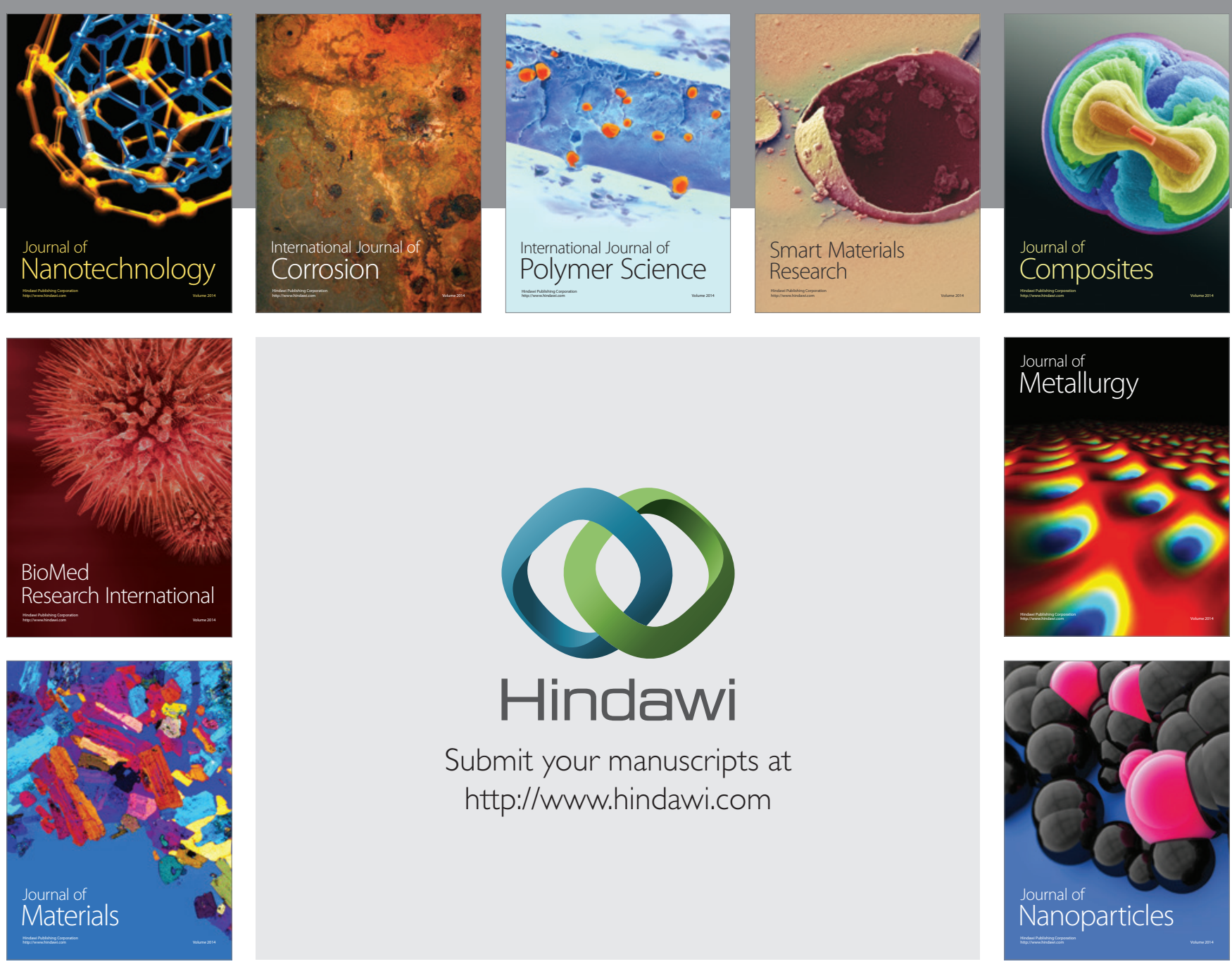

Submit your manuscripts at http://www.hindawi.com
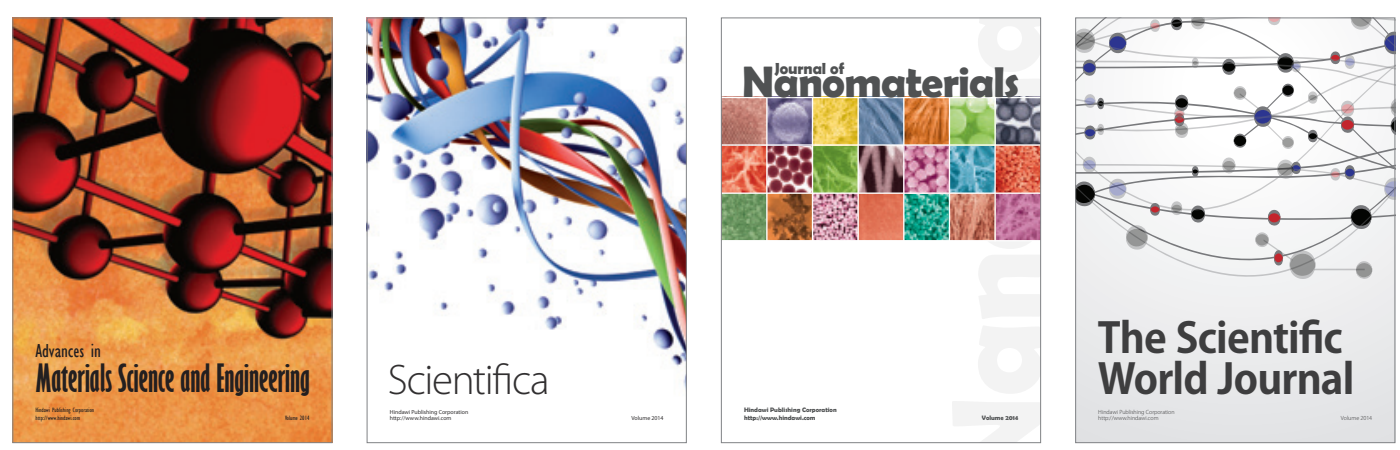

\section{The Scientific World Journal}
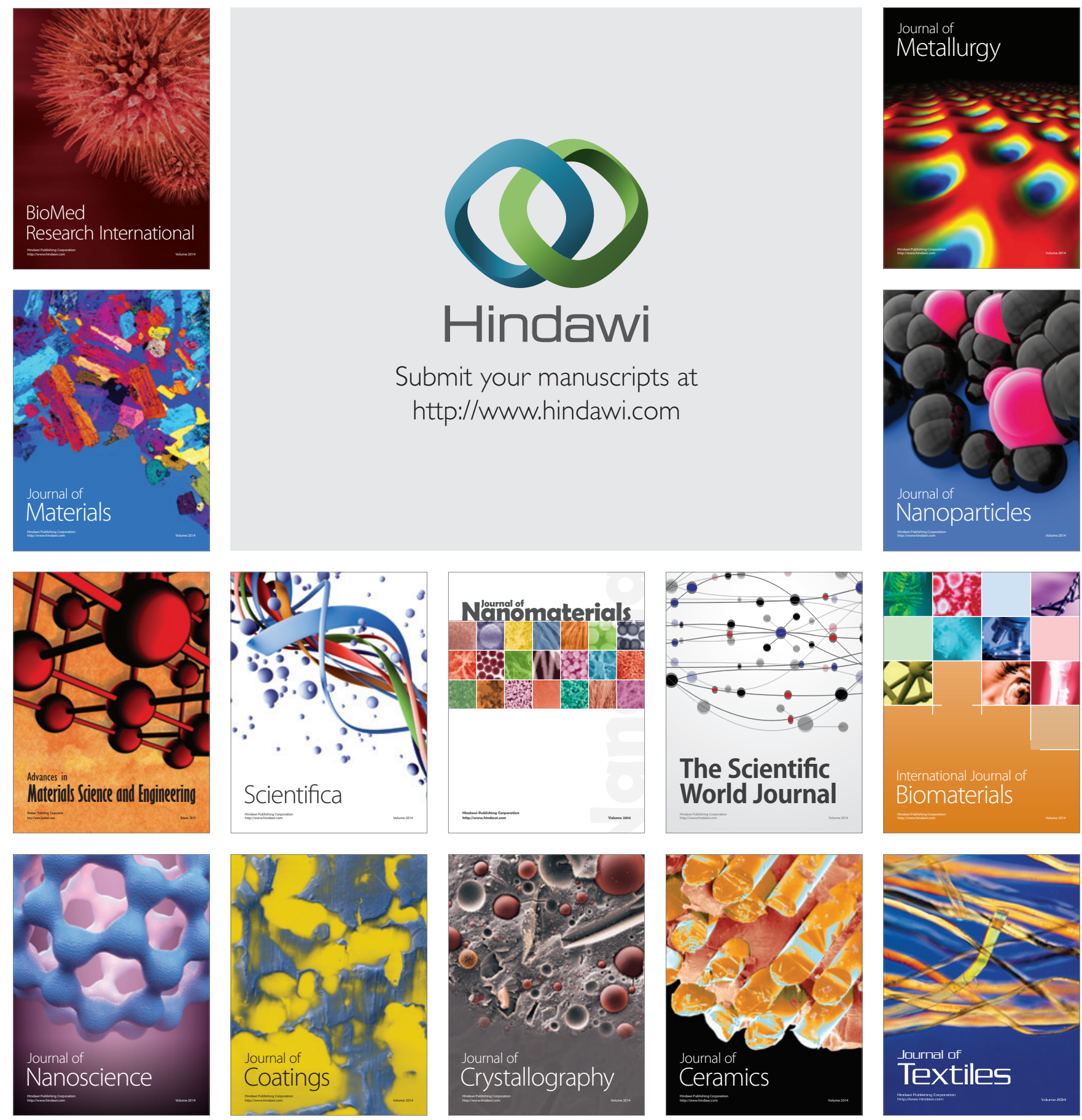